\title{
Experiment on Bare Fiber Stress Fatigue Life Index
}

\author{
Zhiyuan Pan ${ }^{1,}$ a \\ ${ }^{1}$ School of science, Wuhan university of technology huaxia college, No. 589, Guansan Road, Wuhan \\ 430070, China \\ apzy820126@163.com
}

Keywords: Fibre technology; Static; Dynamic; Cyclic; Fatigue.

\begin{abstract}
The optical fiber has been extensively used as a sensor in some special fields. Because the optical fiber exists crackles itself and also produce the micro-crack as well as local damage, the fatigue behavior of the optical fiber become the main problem we care about. This paper presents the experimental study on the fatigue performance of bare fiber under static, dynamic and cyclic load. The corresponding fatigue life index (Stress corrosion index) $\mathrm{n}$ can be obtained, which are 17.14, 11.83 and 5.88, respectively. The experiment data reveal that fatigue life index is related to loading mode and is treated as the indicator of crack propagation resistance of materials. Because dynamic and cyclic loads can induce additional damage, the fatigue life declines dramatically under the same stress levels.
\end{abstract}

\section{Introduction}

As the aerospace structure safety and reliability requirements are continuously improved, the online monitoring and diagnosis of the structure damage have attracted much attention [1]. The strain monitoring of the structure is the significant indicator of its damage degree. And optical fiber Bragg grating sensor, due to its improving properties such as small size, light weight, outstanding anti-jamming capability as well as characteristic of wavelength division multiplex and wavelength encoding, can be used to monitor the strain and the damage of the aviation and aerospace structures. The fiber is the sensory unit of the sensor. Through the fiber embedded in the composite material, the strain, degrees, cracks and the fatigue and damage information of the structure can be measured real-time [2], and then evaluation and prediction can be made on the fatigue failure of the material [2-6].

The optical fiber is also in the state of stress when used as a unit of the sensor. The fatigue performance of the bare fiber in various complex working conditions has become a topic of interest. The failure behavior of the optical fiber is mainly the result of subcritical crack growth due to its brittleness. Therefore the damage mechanism can be analyzed and revealed based on the linear fracture mechanism against the stress intensity in the crack tip area and the laws for the crack growth rate can also be obtained [7-13], which are valuable information for better application of the optical fiber. On the basis of fatigue theories of glass, this paper obtains fatigue indexes at three different loads from experiment to evaluate the failure behaviors of the optical fiber at different loads. Meanwhile, the static fatigue curve is fitted and obtained effectively based on the experimental data for the dynamic fatigue at the three loads.

\section{Experiment}

The optical fiber used in the experiments is produced by Wuhan ChangFei optical fiber and cable Co., LTD. The chemical composite of the external protective coating is the poly aluminum chloride (PAC). The bare optical fiber can be obtained by dissolving PAC from the surface of the optical fiber. The average diameter of the bare optical fiber is $107.62 \mu \mathrm{m}$. Each end of the optical fiber is reinforced by the carbon fiber sheet. And the adhesive is solidified completely by being dried at an oven of $60^{\circ}$ constantly for 24 hours. Specimens and experiment devices are shown in Figure. 1. 
In this paper, the fatigue properties of the bare optical under static, dynamic and cyclic loads have been tested. All the samples are $50 \mathrm{~mm}$ long. The static loading was applied by hanging a weight block at the end of the sample. The blocks weigh $0.85^{\sigma_{b}}, 0.8^{\sigma_{b}}, 0.75^{\sigma_{b}}, 0.7^{\sigma_{b}}$, and $0.65^{\sigma_{b}}$, respectively ( $\sigma_{b}$ 0.0005). The initial hanging time of every sample was recorded and the whole process filmed by computer and camera. Thus, the cracking-time of every sample could be obtained. The dynamic loading was generated by the Instron 5848 testing machine. The uniaxial tension was applied, and the tensile rates were $5 \mathrm{~mm} / \mathrm{min}, 0.5 \mathrm{~mm} / \mathrm{min}, 0.05 \mathrm{~mm} / \mathrm{min}, 0.005 \mathrm{~mm} / \mathrm{min}$, and $0.0005 \mathrm{~mm} / \mathrm{min}$, respectively. The cyclic loading was also generated by the Instron5848 testing machine. The uniaxial tension was chosen in the experiment. The maximum stresses were $0.9^{\sigma_{b}}, 0.85^{\sigma_{b}}, 0.8^{\sigma_{b}}, 0.75^{\sigma_{b}}$, $0.7^{\sigma_{b}}$, and $0.65^{\sigma_{b}}$, respectively. The minimum tensile loading was $5 \mathrm{~N}$ and the frequency $2 \mathrm{~Hz}$ for all the cyclic loads.

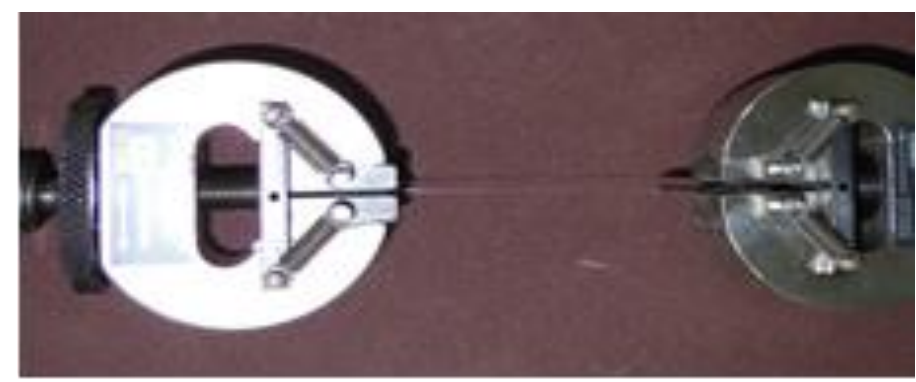

Figure. 1 A specimen and experiment device

\section{Results and Discussion}

Static Fatigue Test. The fatigue property of brittle materials is characterized by the subcritical crack growth $\mathrm{V}$, which can be calculated by the stress intensity factor $\mathrm{KI}$ as:

$$
V=A K_{I}^{n}
$$

where $\mathrm{A}$ is the constant and $\mathrm{n}$ the fatigue life index.

$$
K_{I}=Y \sigma_{a} a^{1 / 2}
$$

where $\mathrm{Y}$ is the crack geometry factor, $\sigma_{a}$ the applied stress, and $a$ the length of the crack.

The value of the material fatigue life can be obtained by substituting the Eq. (2) into Eq. (1) to get the integration when $\sigma_{a}$ is considered as a constant.

The formulation of the fatigue life is written as:

$$
t_{f}=B \cdot \sigma_{c}^{n-2} \cdot \sigma_{a}^{-n}\left[1-\left(\sigma_{a} / \sigma_{c}\right)^{n-2}\right]
$$

Where $\sigma \mathrm{c}$ is the inherent intensity of the material, $\mathrm{B}$ is only related with the material and the working conditions, and can be written as:

$$
B=2 /\left[A Y^{2}(n-2) \cdot K_{I C}{ }^{n-2}\right]
$$

Because $\sigma_{a}$ is smaller than $\sigma_{c}$ and the $\mathrm{n}$ is generally large, the relationship between $\sigma_{a}$ and $\sigma_{c}$ can be obtained. It can be written as: $\left(\sigma_{a} / \sigma_{c}\right)^{n-2}<<1$. The Eq. (3) can be simplified as follows:

$$
t_{f}=B \cdot \sigma_{c}^{n-2} \cdot \sigma_{a}^{-n}
$$

The logarithm has been taken on both sides of the Eq. (3). The formulation can be written as:

$$
\log _{n} \sigma_{a}=-1 / n \cdot \log _{n} t_{f}+1 / n \cdot \log _{n} B+(n-2) / n \cdot \log _{n} \sigma_{c}
$$


The relationship between $\sigma_{a}$ and $\mathrm{tf}$ is linear and the slope is $-1 / \mathrm{n}$. The fatigue life index can be solved by the Eq. (6). The experimental result is shown in the Figure. (2) and the corresponding fatigue life index $n$ is 17.14 .

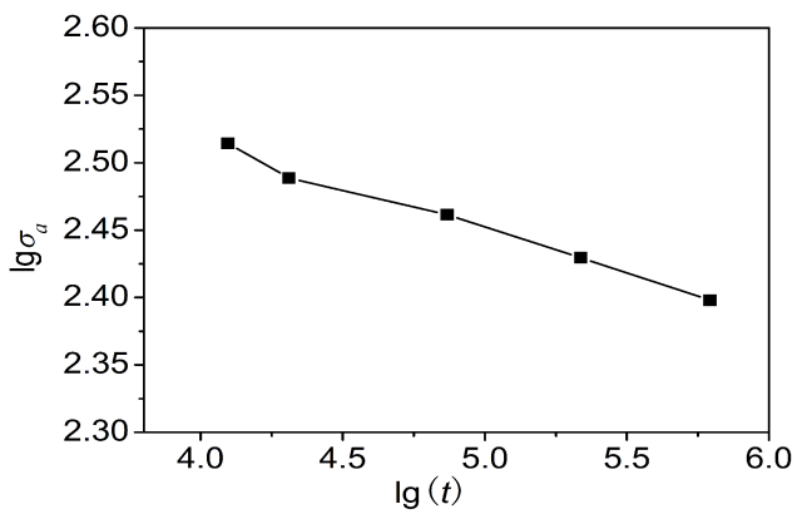

Figure 2. Relation between loading stress and failing time under static fatigue

Dynamic Fatigue Test. The total differential of the stress to the crack length is as follows: $d \sigma / d a=\dot{\sigma} / V$, where $\dot{\sigma}$ is the stress rate. Because the crack growth rate is the power function of the stress intensity factor, it can be written as:

$$
d \sigma / d a=\dot{\sigma} / A K_{I}^{n}=\dot{\sigma} / A Y^{n} \sigma^{n} a^{n / 2}
$$

The Eq. (8) can be obtained by the separation of the variables from the integration.

$$
\sigma_{f}^{n+1}=B(n+1) \dot{\sigma}\left(\sigma_{c}^{n-2}-\sigma_{f}^{n-2}\right)
$$

Because of $\sigma \mathrm{f}<<\sigma \mathrm{c}$, the $\sigma \mathrm{f}$ can be written as:

$$
\sigma_{f}^{n+1}=B(n+1) \dot{\sigma} \sigma_{c}^{n-2}
$$

The logarithm has been taken on both sides of the Eq. (9). The formulation can be written as:

$$
\log _{n} \sigma_{f}=1 /(n+1) \log _{n} \dot{\sigma} \sigma_{c}+1 /(n+1) \log _{n}\left[B(n+1) \sigma_{c}^{n-2}\right]
$$

The loading rate should be the average value of the whole loading process. That is $\sigma=\sigma_{f} / t_{f}$, where $\sigma$ is the loading rate, $\sigma_{f}$ the fracture stress and tf the loading time. So the loading rate should be $127.04 \mathrm{MPa} / \mathrm{sec}, 10.69 \mathrm{MPa} / \mathrm{sec}, 1.42 \mathrm{MPa} / \mathrm{sec}, 0.134 \mathrm{MPa} / \mathrm{sec}, 0.014 \mathrm{MPa} / \mathrm{sec}$, respectively. The values of the test points in the experiment are all the average of the ten samples.

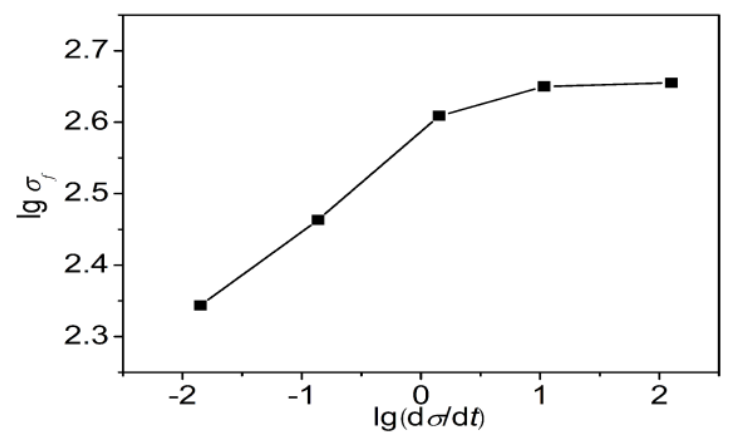

Figure 3. Relation between failing stress and strain rate under dynamic fatigue 
The relationship between $\sigma_{f}$ and $\dot{\sigma}$ is linear and the slope is $1 /(\mathrm{n}+1)$. The fatigue life index can be solved by the Eq. (10). The experimental result is shown in the Figure.(3) and the corresponding stress corrosion index $\mathrm{n}$ is 11.83 .

Cyclic Fatigue Test. The load is the periodic function of time in the cyclic fatigue test. It can be written as: $\sigma=\dot{\sigma}(t)$. The following formula can be obtained through similar mathematical calculation for the constant stress conditions. It can be written as: $\int_{o}^{t_{f}} \sigma^{n}(t) d t=B \cdot \sigma_{c}^{n-2}$. The dimensionless parameter $\mathrm{g}$ is introduced to characterize the types of the stress. And the analytical formula of the parameter $\mathrm{g}$ is as follows:

$$
g=t_{f}^{-1} \int_{o}^{t_{f}}\left[\frac{\sigma(t)}{\sigma_{c h}}\right]^{n} d t
$$

The Eq. (12) can be obtained through the integration into the Eq. (11).

$$
t_{f}=g^{-1} B \sigma_{c}^{n-2} \sigma-n_{c h}{ }^{-n}
$$

Where $\sigma \mathrm{ch}$ is the eigenvalue of $\sigma(\mathrm{t})$. The Eq. (13) can be obtained by the integration into the Eq. (12).

$$
\log _{n} t_{f}=\log _{n}\left(g^{-1} B \sigma_{c}^{n-2}\right)-n \log _{n} \sigma_{c h}
$$

The relationship between $\sigma_{c h}$ and $t_{f}$ is linear and the slope is the fatigue life index $\mathrm{n}$ in double logarithm coordinates. The experimental result of the cyclic fatigue test is shown in the Figure. (4). The maximum value of the cyclic stress is the eigenvalue and the corresponding fatigue life index $n$ is 5.88 .

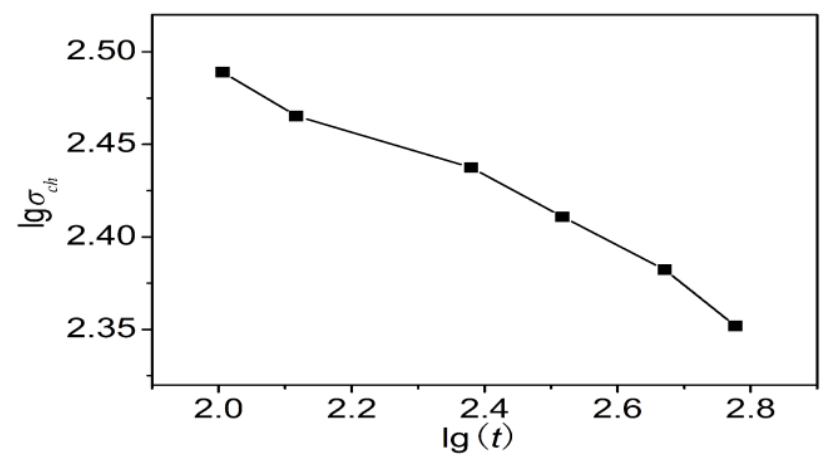

Figure. 4 Relation between loading maximum stress and failing time under cycle fatigue

Effect of the loading conditions on fatigue life index

Reports have suggested that, the displacement is linear increasing with the increase of force in the start of loading, but displacement of optical fiber bobs with the increase of loading after load reaches a relatively high level when the coating separates from the wrap. The core of fiber bears the weight of loading after increasing load, and the core of fiber goes fragile immediately. When the optical fiber is buried in the matrix, we reckon that the separation between the wrap and the coating will not affect sensory property. Hence the mechanical properties of wrap and core of fiber determines whether sensory property of optical fiber is applied.

In the article, we use acetone bubble to wipe off coating of fiber, constrain the both ends and goes on experimental study. The fatigue index of that is gauged under various loading and is used to study the behavior of bare optical fiber in use. The fatigue life indexes under the static fatigue test, dynamic fatigue test and cyclic fatigue test are 17.14, 11.83 and 5.88 respectively. The difference demonstrates that the fatigue life index is strongly dependent on the loading conditions. The fatigue 
life index under the dynamic loading condition declines by one third compared with the index under the static loading condition. And the fatigue life index under the cyclic loading condition declines by one half compared with the index under the dynamic loading condition.

The experimental results reveal that the fatigue life index and the loading condition are not independent of each other. The fatigue life index is treated as the indicator of crack growth resistance, because the predicted value of the material fatigue life increases as the fatigue life index grows. And the stress corrosion index varies with the different loading condition. That means the material fatigue life is different under different loading conditions. The fatigue life under static loading is higher than that under dynamic loading, and that under dynamic loading is higher than under cyclic loading. This should be due to the additional damage existing in the material under the dynamic loading or the cyclic loading conditions. And the additional damage under the cyclic loading condition is more severe than under the dynamic loading conditions

\section{Conclusions}

The fatigue performance subject to static, dynamic and cyclic load of bare fiber has been systematically investigated by experiment. The value of fatigue life index $n$ is closely related to the loading conditions, and it can be the indicator of fatigue damage resistance. The fatigue life declines dramatically under the same stress levels due to the additional damage induced by the dynamic and cyclic load. The results reveal that the subcritical crack growth will occur when the stress intensity factor (KI) is less than the subcritical crack growth factor (KIC). The relationship between the growth rate $(\mathrm{V})$ and the stress intensity factor $(\mathrm{KI})$ is as follows: $\mathrm{V}=\mathrm{AKI}$. The fatigue indexes under the three kinds of loading conditions are 17.68, 11.83 and 5.88, respectively.

\section{Acknowledgements}

The work is supported with scientific research plan guide project B2014278, from Hubei province ministry of education.

\section{References}

[1] Cooper KR, Elster J, Jones M, Kelly RG. IEEE, 2001; 5:847-856.

[2] Leung CKY, Yang ZL, Xu Y, Tong P, Lee SKL. Sens. Actuators, A 2005; 119:336-344.

[3] Suopajarvi P, Lyori V, Nissilae SM, Kopola HK, Johansson R. Opt Eng 1995; 34(9):2587-2591.

[4] Lekakou C, Cook S, Deng Y, Ang TW, Reed GT.Composites Part A 2006; 37:934-938.

[5] Dumitrescu OR, Baker DC, Foster GM, Evans KE. Polym Test 2005; 24:367-375.

[6] Frazao O, Ramos CA, Pinto NMP, Baptista JM, Marques AT. Compos Sci Technol 2005;65:1756-1760.

[7] Lafitte MH, Bunsell AR. J Mater Sci 1982;17:2391-2397.

[8] Dobb MG, Robson RM, Roberts AH. J Mater Sci 1993; 28:785-788.

[9] Parimala HV, Vijayan K. J Mater Sci Lett 1993; 12:99-101.

[10] Hearle JWS, Wong BS. J Mater Sci 1977; 12:2447-2455.

[11]Bunsell AR. J Mater Sci 1975; 10:1300-1308.

[12] Smook J, Pennings J. Colloid Polym Sci 1984; 262:712-722.

[13] Dijkstra DJ, Torf JCM, Pennings AJ. Colloid Polym Sci 1989; 267:866-875. 